\title{
Molecular epidemiologic study of citrin deficiency by screening for four reported pathogenic SLC25A13 variants in the Shaanxi and Guangdong provinces, China
}

\author{
Wei-Xia Lin ${ }^{1 \#}$, Muhammad Rauf Yaqub ${ }^{1 *}$, Zhan-Hui Zhang ${ }^{2}$, Man Mao', Han-Shi Zeng ${ }^{1}$, \\ Feng-Ping Chen ${ }^{3}$, Wei-Ming $\mathrm{Li}^{4}$, Wen-Zhe Cai ${ }^{5}$, Ying-Qiang $\mathrm{Li}^{6}$, Zhi-Yong Tan ${ }^{7}$, Wei Sheng ${ }^{8}$, \\ Zhi-Min $\mathrm{Li}^{9}$, Xiao-Ling Tao ${ }^{10}$, Yuan-Xia Li ${ }^{11}$, Jun-Ping Zhang ${ }^{12}$, Yao-Bin $\mathrm{Han}^{13}$, Yan $\mathrm{Li}^{14}$, \\ Wu-Qiong Duan ${ }^{15}$, Bao-Ni Ye ${ }^{16}$, Ya-Rong Li ${ }^{17}$, Yuan-Zong Song ${ }^{1}$
}

${ }^{1}$ Department of Pediatrics, the First Affiliated Hospital, Jinan University, Guangzhou, China; ${ }^{2}$ Clinical Medicine Research Institute, the First Affiliated Hospital, Jinan University, Guangzhou, China; ${ }^{3}$ Department of Laboratory Science, the First Affiliated Hospital, Jinan University, Guangzhou, China; ${ }^{4}$ Department of Pediatrics, Maternal and Child Health Hospital of Qingyuan City, Qingyuan, China; ${ }^{5}$ Department of Internal Medicine, Central Hospital of Shantou City, Shantou, China; ${ }^{6}$ Department of Internal Medicine, Maternal and Child Health Hospital of Yunfu City, Yunfu, China; ${ }^{7}$ Department of Pediatrics, Maternal and Child Health Hospital of Shaoguan City, Shaoguan, China; ${ }^{8}$ Department of Pediatrics, Weinan First Hospital, Weinan, China; ${ }^{9}$ Department of Pediatrics, San Er Ling Yi Hospital, Hanzhong, China; ${ }^{10}$ Department of Pediatrics, Xianyang Rainbow Hospital (Xianyang Children's Hospital), Xianyang, China; ${ }^{11}$ Department of Pediatrics, Yan'an University Hospital, Yan'an, China; ${ }^{12}$ Clinical laboratory, Qishan County Hospital, Qishan, China; ${ }^{13}$ Department of Infectious Diseases, Shenmu Hospital of Northwestern University, Shenmu, China; ${ }^{14}$ Department of Pediatrics, Zhashui County Hospital, Zhashui, China; ${ }^{15}$ Department of Pediatrics, Ankang Central Hospital, Ankang, China; ${ }^{16}$ Department of Pediatrics, Southern Campus of Tongchuan People's Hospital, Tongchuan, China; ${ }^{17}$ The Third Department of Infectious Diseases, Xi'an Children's Hospital, Xi'an, China

Contributions: (I) Conception and design: YZ Song; (II) Administrative support: YZ Song; (III) Provision of study materials or patients: FP Chen, WM Li, WZ Cai, YQ Li, ZY Tan, W Sheng, ZM Li, XL Tao, YX Li, JP Zhang, YB Han, Y Li, WQ Duan, BN Ye, YR Li, YZ Song; (IV) Collection and assembly of data: WX Lin, MR Yaqub, ZH Zhang, M Mao, HS Zeng; (V) Data analysis and interpretation: WX Lin, MR Yaqub; (VI) Manuscript writing: All authors; (VII) Final approval of manuscript: All authors.

\#These authors contributed equally to this work.

Correspondence to: Yuan-Zong Song. Department of Pediatrics, the First Affiliated Hospital, Jinan University, Guangzhou 510630, China. Email: songyuanzong@vip.tom.com or songyuanzong@hotmail.com; Ya-Rong Li. The Third Department of Infectious Diseases, Xi'an Children’s Hospital, Xi’an 710002, China. Email: lyr640101@163.com.

Background: Citrin deficiency $(\mathrm{CD})$ is an autosomal recessive disease resulting from biallelic mutations of the SLC25A13 gene. This study aimed to investigate the molecular epidemiological features of CD in the Guangdong and Shaanxi provinces of China.

Methods: A total of 3,409 peripheral blood samples from Guangdong and 2,746 such samples from Shaanxi province were collected. Four prevalent SLC25A13 mutations NG_012247.2 (NM_014251.3): c.852_855del, c.1638_1660dup, c.615+5G>A, and c.1751-5_1751-4ins(2684) were screened by using the conventional polymerase chain reaction (PCR)/PCR-restriction fragment length polymorphism and newly-developed multiplex PCR methods, respectively. The mutated SLC25A13 allele frequencies, carrier frequencies, and CD morbidity rates were calculated and then compared with the Chi-square and Fisher's exact tests.

Results: The mutations were detected in 68 out of 6,818 SLC25A13 alleles in Guangdong and 29 out of 5,492 alleles in the Shaanxi population. The carrier frequencies were subsequently calculated to be $1 / 51$ and $1 / 95$, while the CD morbidity rates were $1 / 10,053$ and $1 / 35,865$, in the 2 populations, respectively. When compared with the Shaanxi population, Guangdong exhibited a higher frequency of mutated SLC25A13 allele $\left(68 / 6,818\right.$ vs. $\left.29 / 5,492, \chi^{2}=8.570, \mathrm{P}=0.003\right)$ in general, with higher c.852_855del $(54 / 6,818 v s .13 / 5,492$,

^ ORCID: 0000-0003-2928-250X. 
$\left.\chi^{2}=17.328, \mathrm{P}=0.000\right)$ but lower c.1751-5_1751 -4ins(2684) (2/6,818 vs. 9/5,492, $\left.\mathrm{P}=0.015\right)$ allele frequencies. The distribution of c. $615+5 \mathrm{G}>\mathrm{A}$ and c.1638_1660dup between the 2 provinces, as well as all 4 prevalent mutations among different geographic regions within the 2 provinces, did not differed significantly.

Conclusions: Our findings depicted the CD molecular epidemiological features in Guangdong and Shaanxi populations, providing preliminary but significant laboratory evidences for the subsequent CD diagnosis and management in the 2 provinces of mainland China.

Keywords: Citrin deficiency; epidemiology; Shaanxi; Guangdong

Submitted Feb 07, 2021. Accepted for publication Apr 19, 2021.

doi: $10.21037 / \mathrm{tp}-21-58$

View this article at: http://dx.doi.org/10.21037/tp-21-58

\section{Introduction}

Citrin deficiency (CD) is an autosomal recessive genetic metabolic disease arising from a functional defect of citrin, the aspartate/glutamate carrier isoform 2 in the mitochondrial inner membrane of the hepatocyte in humans $(1,2)$. The causative gene SLC25A13 is localized at chromosome $7 \mathrm{q} 21.3$ and spans about $200 \mathrm{~kb}$ in length with 18 exons and 17 introns (3). Citrin functions to exchange aspartate synthesized by the mitochondrial matrix with glutamate and a proton in the cytoplasm of the hepatocyte, playing distinct roles in the urea cycle, malate-aspartate shuttle and gluconeogenesis (4-7).

From July 2005 to August 2020, our team diagnosed 458 CD patients by $S L C 25 A 13$ gene analysis from 29 different provinces, municipalities, and autonomous regions of China, and the 4 mutations of NG_012247.2 (NM_014251.3): c.852_855del, c.1638_1660dup, c.615+5G>A, and c.1751-5_1751-4ins(2684) were at the top of the list, accounting for $82.9 \%$ of all mutated alleles [(8) partial data unpublished]. In 2014, we screened the above 4 prevalent mutations using HybProbe assay and High Resolution Melting Assay in 2,428 healthy subjects from 9 cities of Guangdong, a province in the southeast of mainland China (9); this revealed a carrier frequency of $1 / 47$ and a theoretical morbidity of $1 / 8,800$. However, $64.2 \%(1,558 / 2,428)$ of the research participants were from 5 cities of the Pearl River Delta area, which only accounted for about $36.5 \%$ (38/104 million) of permanent resident populations in Guangdong, while the imbalance of the sample sizes might have led to a bias in the results.

According to the findings of human genetic diversity, the Chinese population can be divided into 2 large groups from the north to the south along the boundary of the Yangtze River $(10,11)$. The carrier frequency of
SLC25A13 mutations in the north (1/940) was found to be significantly lower than that in the south (1/48) (12); however, the prevalent mutation c.1751-5_1751-4ins(2684) was uncovered in 2008 (13), and thus was not included in the previous study. Notably, most research participants in previous studies were from the middle and coastal areas of China, and an epidemiologic study of CD in the northwest China has not yet been conducted.

Shaanxi Province is located in northwest China and adjoins the other 8 provinces. In this study, the 4 prevalent SCL25A13 mutations were screened using 981 additional peripheral blood samples collected from 4 peripheral cities in Guangdong and 2,746 of these samples from different cities in Shaanxi. The carrier frequencies of the prevalent mutations and the morbidity rates of CD were then calculated and their distribution was compared.

We presented the following article in accordance with the STROBE reporting checklist (available at http://dx.doi. org/10.21037/tp-21-58).

\section{Methods}

\section{Participants}

According to the latest official data by the National Bureau of Statistics, China, the permanent resident populations of the provinces of Guangdong and Shaanxi at the time of this study were 104 and 37 million, respectively (http://www. stats.gov.cn/tjsj/tjgb/rkpcgb/). In order to achieve statistical significance, the sample sizes were calculated to be at least 1,953 and 695 on the basis of the estimated carrier rates of 1/48 in Guangdong and 1/940 in Shaanxi by using the online sample size calculator EPITOOLS (https://epitools. ausvet.com.au/twoproportions), with a confidence level of $95 \%$ and the power of 0.99 . In the case of sample loss and 


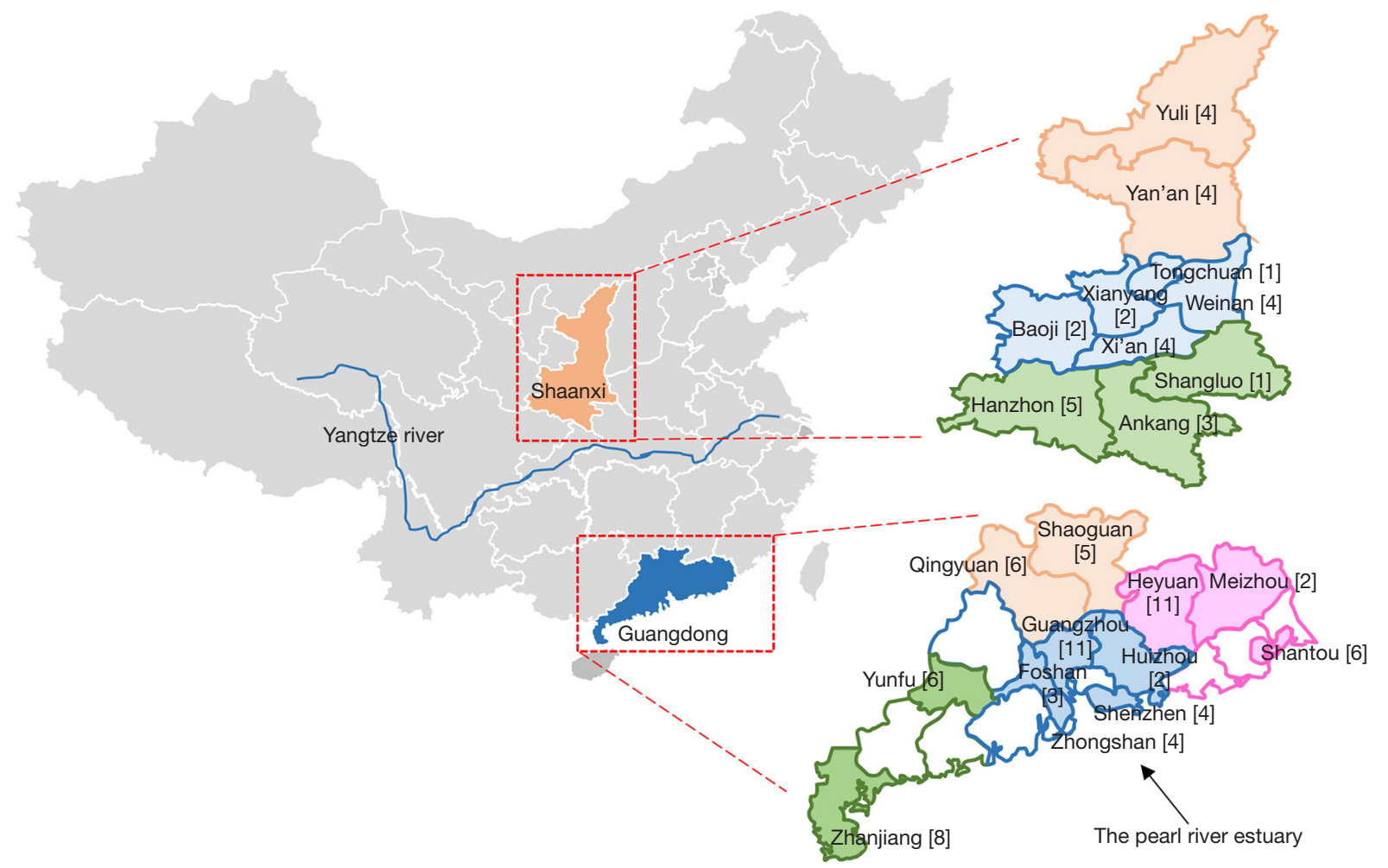

Figure 1 Geographic distribution of the mutated SLC25A13 alleles in different cities of the Guangdong and Shaanxi provinces of China. Guangdong Province comprises of 4 regions: north (orange), west (green), east (pink), and the Pearl River Delta area (blue). Shaanxi Province comprises of 3 areas: north (light orange), center (light blue) and south (light green). The mutated SLC25A13 alleles from different cities are marked in parentheses. This figure was generated by means of the software WPS Office PowerPoint 2019. The base map was created by incrementally assembling the outlines of the Chinese administrative regions, which could be downloaded via the URL link http:// www.900ppt.com/.

a higher carrier rates of CD in Shaanxi, more participants were added to enlarge the sample size. A computer-based randomization was used to select samples from each of the regions. The number of samples from each region was in accordance with the population distribution.

Besides the 2,428 samples in our previous study (9), we collected an additional 981 blood samples for health examination from 4 peripheral cities (Heyuan, Shaoguan, Shantou, and Yunfu) in Guangdong and 2,746 of these samples from 10 cities (Yan'an, Yulin, Weinan, Xianyang, Baoji, Xi'an, Tongchuan, Hanzhong, Shangluo, and Ankang) in Shaanxi between June 2016 and December 2018 (Figure 1).

This study was conducted in accordance with the Declaration of Helsinki (as revised in 2013) and was approved by the Medical Ethics Committee of the First Affiliated Hospital, Jinan University, Guangdong,
China (NO.: KY-2019-052, 2015-038, 2014-004). Participants were genotyped retrospectively using blood samples previously collected for the purpose of health examination. The data related to individual identification were anonymized during the entire study process. Therefore, informed consent was waived.

\section{Genetic analysis}

Genomic DNA was purified from the peripheral blood samples. The mutations c.1638_1660dup and c.615+5G>A were detected using established polymerase chain reaction (PCR) and PCR-restriction fragment length polymorphism methods, as in previous publications $(12,14)$. The mutations c.852_855del and c.1751-5_1751-4ins(2684) were screened using newly-developed multiplex PCR approaches as described below. 
Four primers, MuI-3NF (5'-TGGCAATTTGAATA ACATCAGATGAC-3', forward), MuI-NR1-AP2 (5'-AC TATGGGGCCGTTCAATGTCTGCTAAGGTCA TA-3', reverse), MuI-AF4-AP2 (5'-ACGCGTGTGTTG TTTTTCCCCTACAGACGAC-3', forward) and MuI9AR (5'-CAGTGACACCAACACGGGATTCT-3', reverse), were designed for the multiplex PCR amplification of mutation c.852_855del (Figure 2A); the optimal concentrations of the 4 primers were set at a ratio of 1:1:0.5:0.5, respectively. The PCR cycling condition included an initial denaturation step of $94^{\circ} \mathrm{C}$ for $5 \mathrm{~min}, 35$ cycles of denaturation at $94^{\circ} \mathrm{C}$ for $30 \mathrm{sec}$, annealing at $57^{\circ} \mathrm{C}$ for $30 \mathrm{sec}$ and extension at $72^{\circ} \mathrm{C}$ for $1 \mathrm{~min}$, as well as a final extension at $72{ }^{\circ} \mathrm{C}$ for $7 \mathrm{~min}$.

For the multiplex PCR amplification of mutation c.1751-5_1751-4ins(2684), 1 forward primer MuXIX-2UF (5'-GCCAAACCACTTACAGCGGAGT-3') along with 2 reverse primers MuXIX-2UR (5'-TTATGACAGAGAGCA GCACTGGTTC-3') and MuXIX-1R (5'-TCCCTACGAC AACAGAGCATTAGC-3') were designed (Figure 2B), and their optimal concentrations were set at a ratio of 5-6:4-5:1, respectively. The temperature condition was as follows: $94{ }^{\circ} \mathrm{C}$ for $5 \mathrm{~min}$, followed by 35 cycles of $94{ }^{\circ} \mathrm{C}$ for $30 \mathrm{sec}$, $60{ }^{\circ} \mathrm{C}$ for $30 \mathrm{sec}$ and $72{ }^{\circ} \mathrm{C}$ for $40 \mathrm{sec}$, as well as a final extension step of $72{ }^{\circ} \mathrm{C}$ for $7 \mathrm{~min}$.

\section{Calculation of the allele frequencies, carrier frequencies and $C D$ morbidity rates}

Geographically, Guangdong Province includes the Pearl River Delta (Guangzhou, Shenzhen, Foshan, Huizhou, and Zhongshan), along with northern (Qingyuan and Shaoguan), eastern (Meizhou, Heyuan, and Shantou), and western (Zhanjiang and Yunfu) regions (Figure 1); meanwhile, Shaanxi consists of northern (Yulin and Yan'an), central (Weinan, Xi'an, Xianyang, Baoji, and Tongchuan), and southern (Hanzhong, Ankang, and Shangluo) areas (Figure 1).

The mutated SLC25A13 allele frequencies, carrier frequencies and CD morbidity rates in different areas were calculated based on the Hardy-Weinberg equilibrium. The genotypes $A A$ (healthy individual), $A B$ (carrier) and $B B$ (patient) of a biallelic genetic marker were expected to have the relative frequencies of $p^{2}, 2 p q$ and $q^{2}$, with $p$ and $q$ being the $A$ (wild type) and $B$ (mutant) allele frequency, respectively; thus, $p+q=1$. The values of $2 p q$ and $q^{2}$ represented as the carrier frequency of SLC25A13 variants and CD morbidity rate, respectively $(15,16)$.

\section{Statistical analysis}

By using the statistical software SPSS version 23.0 (IBM Corp., Chicago, IL, USA), the distributions of the 4 SLC25A13 variants were compared via Chi-square and Fisher's exact tests among different geographic regions, with a $\mathrm{P}$ value $<0.05$ indicating statistical significance.

\section{Results}

\section{Mutation screening}

To evaluate the reliability of the new PCR methods in this study, the 4 prevalent SLC25A13 mutations were detected in the 200 samples of Qingyuan city, and the results were completely consistent with those in the previous study by using HybProbe assay and HRMA approaches (9).

This study detected 13 individuals with the c.852_855del, 1 with the c.1638_1660dup, and 4 with the c. $615+5 \mathrm{G}>\mathrm{A}$ mutation in the 981 additional samples from Guangdong (Table 1). Therefore, together with the screening findings in our previous study of 2,428 samples (9), a total of 68 mutant SLC25A13 alleles were detected in 6,818 independent alleles (3,409 samples). Additionally, a total of 29 mutated SLC25A13 alleles in 28 individuals were detected among the 5,492 alleles from Shaanxi. Among them, there were 13, 9, 5, and 2 alleles bearing c.852_855del, c.1751-5_17514ins(2684), c.1638_1660dup, and c.615+5G>A, respectively (Table 2). Of note, 1 individual from Xi'an was identified as a homozygote of the c.852_855del mutation, who was apparently healthy but with a specific fondness for highprotein food.

\section{Carrier frequencies and theoretical morbidity rates}

The mutated SLC25A13 allele frequencies in the Guangdong and Shaanxi were $0.997 \%(68 / 6,818)$ and $0.528 \%(29 / 5,492)$, the carrier frequencies were $1 / 51$ and $1 / 95$, and the theoretical morbidity rates were $1 / 10,053$ and $1 / 35,865$, respectively. According to the latest population data and the above theoretical morbidity rates in the Guangdong and Shaanxi provinces, it was estimated that at least 10,345 and 1,032 CD patients were distributed in the different cities of these 2 provinces, respectively (Table 2).

The mutated alleles, carrier frequencies, and morbidity 


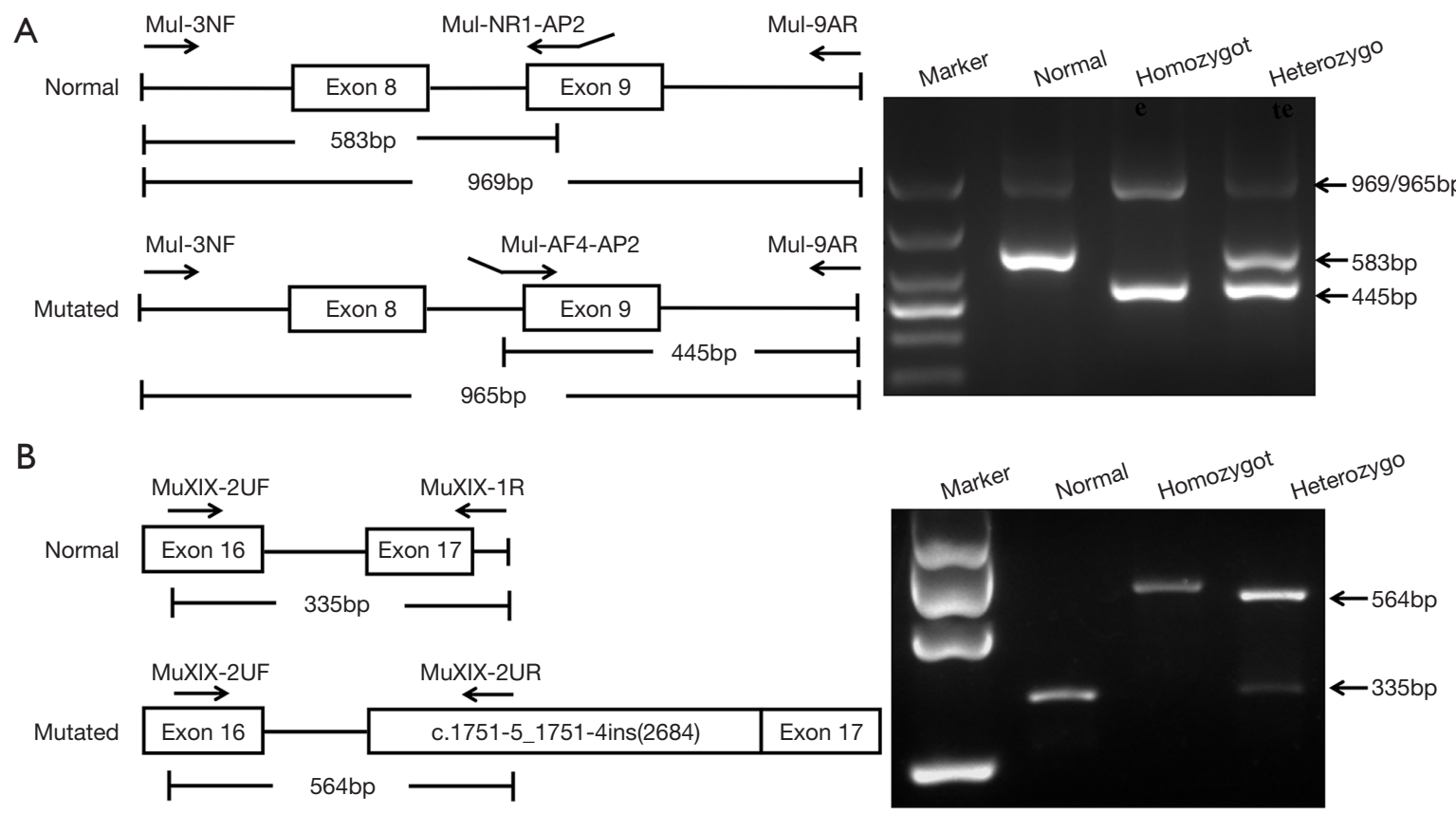

C Normal-969bp GCTTGTGTTTGTTTTTCCCCTACAGACGTATGACCTTAGCAGACATTGÄ̈CGGATTGCTCCTCTGGA A G Mutated-965Bp

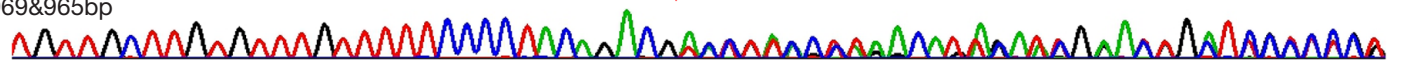

AT T T GT T GCTTGIGTTTGTTTTTCCCCTACAGACGTATGACCTTAGCAGACATTGAACGGCCCCATAGT Normal-583bp

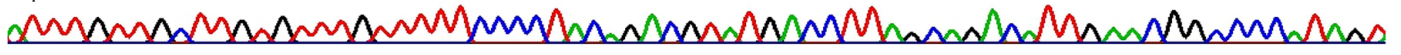 c.852_855del \\ Mutated-445bp \\ TTTCCCCTACAGACG

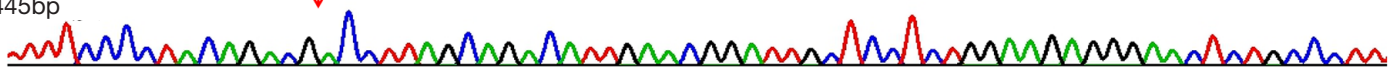 \\ D

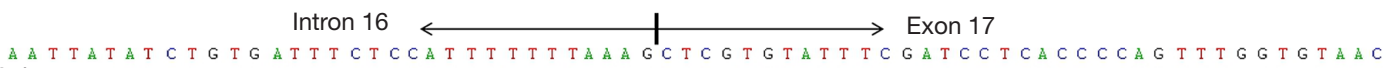

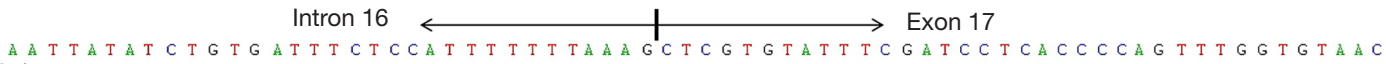 Normal-335bp \\ monmm

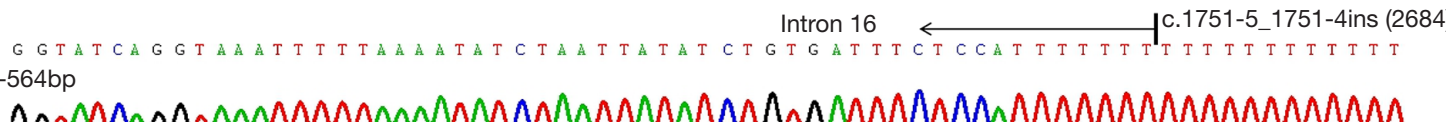 \\ Mutated-564bp}

Figure 2 Novel approaches developed for the screening of the mutations c.852_855del and c.1751-5_1751-4ins(2684). (A) On c.852_855del screening, the wild-type SLC25A13 genotype exhibited 2 bands of $583 \mathrm{bp}$ and $969 \mathrm{bp}$ in size, the homozygote showed 2 bands of 445 bp and $965 \mathrm{bp}$, while the heterozygote showed all 4 bands. In this figure, the 2 bands of $965 \mathrm{bp}$ and $969 \mathrm{bp}$ in sizes represent the PCR products with and without the mutation c.852_855del, respectively. (B) PCR products of the wild-type and homozygote of c.1751-5_1751-4ins(2684) only exhibited a band of $335 \mathrm{bp}$ and $564 \mathrm{bp}$, respectively, while that of the heterozygote presented with both bands. (C) Sanger sequencing of PCR products of c.852_855del. (D) Sanger sequencing of PCR products of c.1751-5_1751-4ins(2684). 
Table 1 Distribution of the 4 prevalent SLC25A13 mutations in different areas of Guangdong Province

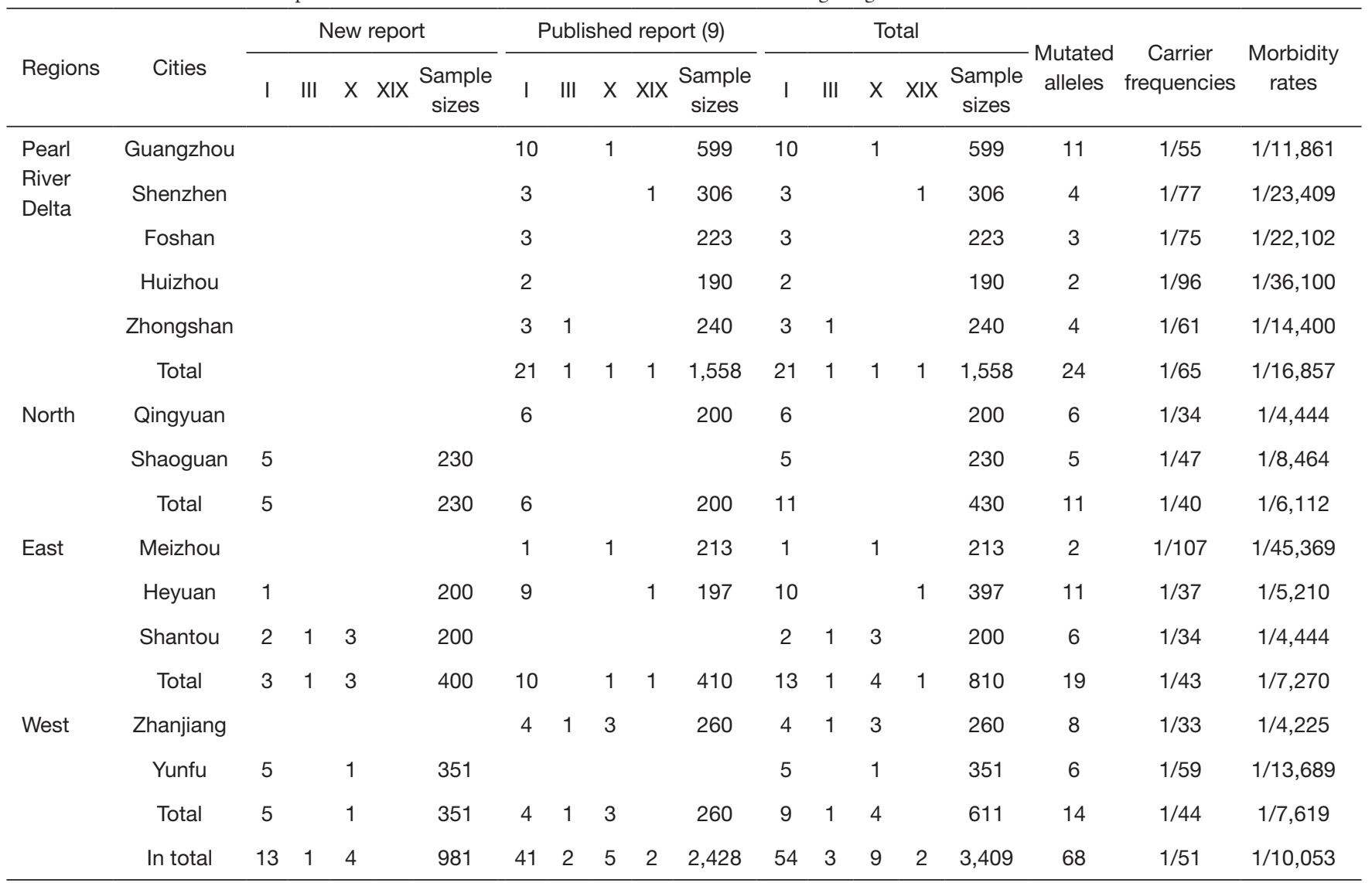

I: c.852_855del; III: c.1638_1660dup; X: c.615+5G>A; XIX: c.1751-5_1751-4ins(2684).

rates among different administrative cities and geographic regions were shown in Tables 2 and 3.

\section{Distribution comparisons of the prevalent SLC25A13 mutations among different geographic regions}

The distribution comparison of the 4 prevalent mutations revealed that Guangdong had a higher mutated SLC25A13 allele frequency than did the Shaanxi population $(68 / 6,818$ vs. $\left.29 / 5,492, \chi^{2}=8.570, \mathrm{P}=0.003\right)$. The mutated allele frequency of c.852_855del was higher in the Guangdong population as compared to that of Shaanxi $(54 / 6,818 \mathrm{vs}$. $\left.13 / 5,492, \chi^{2}=17.328, \mathrm{P}=0.000\right)$ while that of $\mathrm{c} .1751-5 \_1751$ 4ins(2684) was lower $(2 / 6818$ vs. $9 / 5492, \mathrm{P}=0.015)$. The distribution of c. $615+5 \mathrm{G}>\mathrm{A}$ and c.1638_1660dup between the 2 provinces, as well as all 4 prevalent mutations among different geographic regions within the 2 provinces, did not differed significantly $(\mathrm{P}>0.05)$.

\section{Discussion}

Melting curve analysis, including the combination of HybProbe assay and High Resolution Melting Assay, has proven to be a convenient and rapid method for the screening of the 4 prevalent SLC25A13 mutations (9,17-19), but it is technically complex and expensive. Multiplex-PCR consists of multiple sets of primers in a single PCR mixture. By simultaneously amplifying more than 1 fragment in the same reaction, 1-step multiplex PCR was more efficient than simplex PCR $(20,21)$. The c.852_855del and c.17515_1751-4ins(2684) mutations are a 4-base deletion in exon 9 and an about 3 -kb insertion in intron 16 of the SLC25A13 gene, respectively, and have been screened by the newly developed multiplex PCR methods. As shown in Figure 2, 
Table 2 Distribution of the 4 prevalent SLC25A13 mutations in different areas of Shaanxi Province

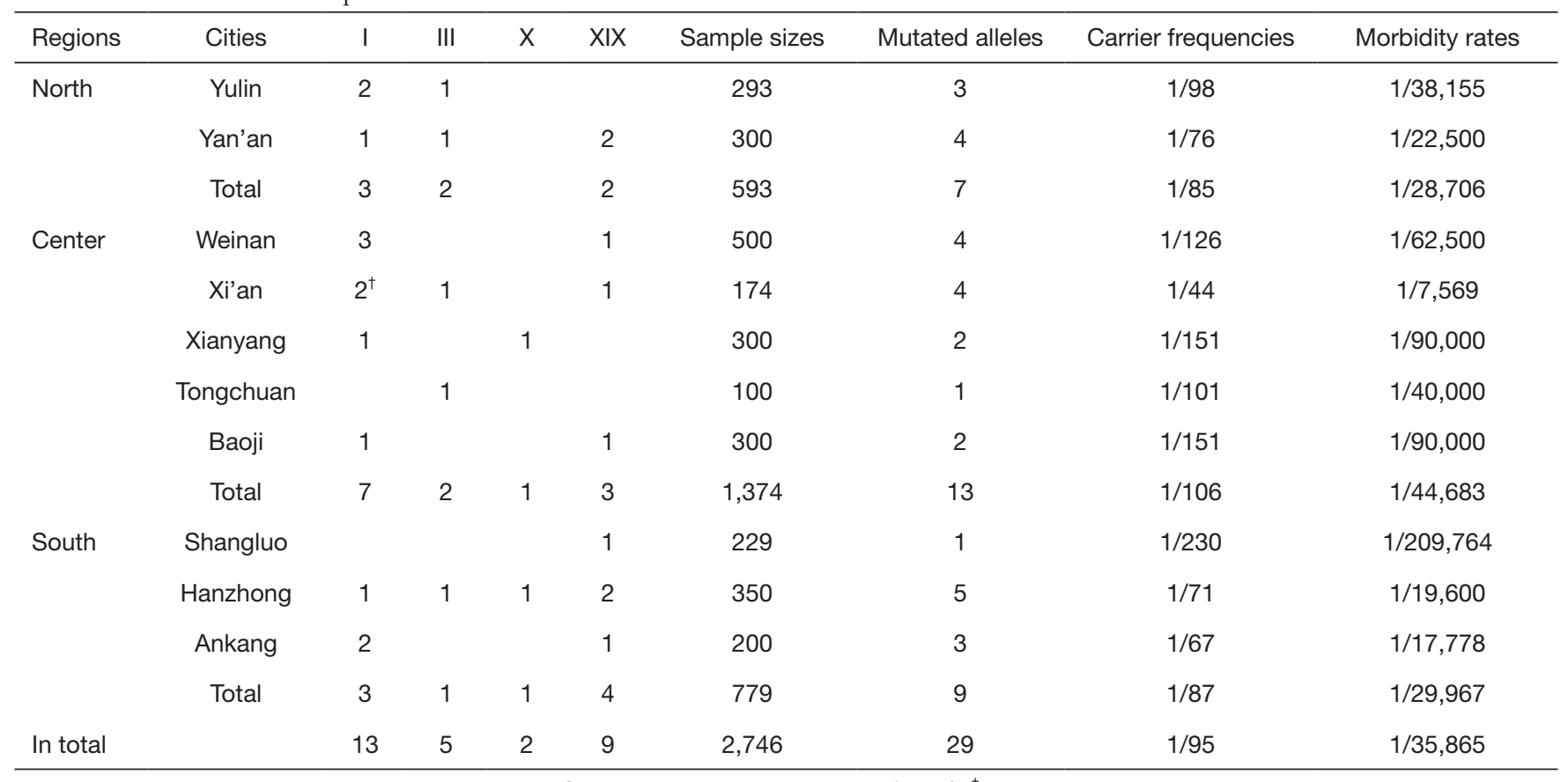

I: c.852_855del; III: c.1638_1660dup; X: c.615+5G>A; XIX: c.1751-5_1751-4ins(2684). ' : Homozygous for the c.852_855del mutation.

Table 3 Comparison of the distribution of the 4 prevalent SLC25A13 mutations in the Guangdong and Shaanxi provinces

\begin{tabular}{lcccc}
\hline Mutations & Guangdong $(n=6,818)$ & Shaanxi $(n=5,492)$ & $\chi^{2}$ & P value \\
\hline I & 54 & 13 & 17.328 & 0.000 \\
III & 3 & 5 & - & 0.480 \\
X & 9 & 2 & - & 0.126 \\
XIX & 2 & 9 & 8.570 & 0.015 \\
Total & 68 & 29 & 0.003 \\
\hline
\end{tabular}

In this table, I, III, X and XIX represent the mutations c.852_855del, c.1638_1660dup, c.615+5G>A, and c.1751-5_1751-4ins(2684), respectively.

with $2-3$ sets of primers in the same PCR reaction, clear and distinguishable amplified DNA fragments covering the wild-type and mutated SLC25A13 alleles were obtained. The combination of conventional PCR/PCR-restriction fragment length polymorphism and newly developed multiplex PCR methods for the detection of 4 prevalent mutations proved to be feasible in a large-scale population analysis in the present study.

In this study, the 4 prevalent SLC25A13 mutations were screened in the 981 new samples from 4 peripheral cities in Guangdong provinces. The sample size was expanded from 2,428 to 3,409 , and the proportions in the
4 geographic regions of Guangdong were thereby made more representative. The 68 carriers identified thus far demonstrated a carrier rate of $1 / 51$ and a theoretical CD morbidity of $1 / 10,053$ in this population, which decreased slightly but might have been more precise than that found in our previous study (9). Data from our previous study showed the mutation carrier rate in the peripheral cities $(1 / 34)$ to be significantly higher than that in the Pearl River Delta area (1/60). However, when more peripheral cities were enrolled in this study, no significant difference of the carrier rate was found between the 2 areas. The reasonable enlargement of the study population minimized the bias 
of the molecular epidemiology study and increased the screening reliability, providing more accurate laboratory evidences for the evaluation of CD effect on the Guangdong population.

For the first time, this study identified 29 mutated SLC25A13 alleles in the 2746 healthy residents in Shaanxi, a province in the northwest China, with a carrier frequency $1 / 95$ and a CD theoretical morbidity rate of about $1 / 35,865$. Surprisingly, the mutated SLC25A13 allele frequency in the Shaanxi population was higher $(29 / 5,492$ vs. $\left.1 / 1,880, \chi^{2}=7.792, \mathrm{P}=0.005\right)$ than that previously found in north China (Liaoning, Beijing, Hebei, Shandong, and Henan) (12), suggesting that the CD prevalence might be underestimated at least in northwest China. Among 4 prevalent mutations, c.852_855del (44.82\%) and c.17515_1751-4ins(2684) (31\%) accounted for almost $75 \%$, suggesting that when performing CD genetic testing, analysis of these 2 mutations should be mandatory in this province. Statistical analysis within the present study discovered homogenous distribution of the prevalent SLC25A13 mutations among different geographic regions in Shaanxi, and hence different molecular targeting might be unnecessary for CD diagnosis in Shaanxi Province.

The distribution comparison revealed that the Guangdong population had a significantly higher mutated SLC25A13 allele frequency than that of Shaanxi, supporting the notion that different geographic distribution of SLC25A13 gene mutations exist in China. It is worth noting that the mutated allele frequency of c.852_855del was significantly higher while that of c.1751-5_1751-4ins(2684) was lower in Guangdong compared to Shaanxi. It has been reported that modern humans colonized East Asia via southern and northern routes on both sides of the Himalayas $(22,23)$. In this study, the 2 prevalent mutations with a different geographic distribution might be attributed to the genetic flow which occurred between distinct founding populations, and their relatively higher frequency could be explained as a result of the founder effect. Actually, the c.852_855del and c.1751-5_1751-4ins(2684) mutations are relatively frequent in East Asia (13,18,19,24-26), with the former being reported as having originated around the Guangxi and Yunnan areas in south China (12) and the latter exhibiting high allele frequency $(33-45.5 \%)$ in Korean $(13,26)$, which is located in the northeast of the Asian continent.

Some limitations in this study should be addressed. Firstly, only focusing on the 4 prevalent mutations might have overlooked other pathogenic SLC25A13 variants in general human populations. Secondly, there might be sampling bias in the 2 provinces. The proportions of research participants in different regions were similar but not identical with the population distribution. Therefore, further epidemiology study focusing on more SLC25A13 variants and with larger sample sizes from the 2 provinces is in need.

\section{Conclusions}

This study highlighted the feasibility of the combination of the conventional PCR/PCR-restriction fragment length polymorphism and the newly developed multiplex PCR methods for the detection of 4 prevalent SLC25A13 mutations in a large-scale population. The findings clarify the molecular epidemiological features of CD in Guangdong and Shaanxi populations, providing preliminary but nonetheless significant laboratory evidence for subsequent CD diagnosis and management in these 2 provinces in mainland China.

\section{Acknowledgments}

The wordings of the main text, figures and tables have been checked by the AME Editing Service (http://editing. amegroups.cn/\#editing).

Funding: This work was supported by the National Natural Science Foundation of China [grant numbers 81570793, 81741080, 81670813, and 81974057] and Natural Science Foundation of Guangdong Province [grant number 2016A030313099].

\section{Footnote}

Reporting Checklist: The authors have completed the STROBE reporting checklist. Available at http://dx.doi. org/10.21037/tp-21-58

Peer Review File: Available at http://dx.doi.org/10.21037/tp21-58

Data Sharing Statement: Available at http://dx.doi. org/10.21037/tp-21-58

Conflicts of Interest: All authors have completed the ICMJE uniform disclosure form (available at http://dx.doi. org/10.21037/tp-21-58). The authors have no conflicts of interest to declare.

Ethical Statement: The authors are accountable for all 
aspects of the work in ensuring that questions related to the accuracy or integrity of any part of the work are appropriately investigated and resolved. The study was conducted in accordance with the Declaration of Helsinki (as revised in 2013) and was approved by the Medical Ethics Committee of the First Affiliated Hospital, Jinan University, Guangdong, China (NO.:KY-2019-052, 2015-038, 2014004). Participants were genotyped retrospectively using blood samples previously collected for the purpose of health examination. The data related to individual identification were anonymized during the entire study process. Therefore, informed consent was waived.

Open Access Statement: This is an Open Access article distributed in accordance with the Creative Commons Attribution-NonCommercial-NoDerivs 4.0 International License (CC BY-NC-ND 4.0), which permits the noncommercial replication and distribution of the article with the strict proviso that no changes or edits are made and the original work is properly cited (including links to both the formal publication through the relevant DOI and the license). See: https://creativecommons.org/licenses/by-nc-nd/4.0/.

\section{References}

1. Palmieri F. Mitochondrial transporters of the SLC25 family and associated diseases: a review. J Inherit Metab Dis 2014;37:565-75.

2. Palmieri F, Monné M. Discoveries, metabolic roles and diseases of mitochondrial carriers: A review. Biochim Biophys Acta 2016;1863:2362-78.

3. Kobayashi K, Sinasac DS, Iijima M, et al. The gene mutated in adult-onset type II citrullinaemia encodes a putative mitochondrial carrier protein. Nat Genet 1999;22:159-63.

4. Thangaratnarajah C, Ruprecht JJ, Kunji ER. Calciuminduced conformational changes of the regulatory domain of human mitochondrial aspartate/glutamate carriers. Nat Commun 2014;5:5491.

5. Amoedo ND, Punzi G, Obre E, et al. AGC1/2, the mitochondrial aspartate-glutamate carriers. Biochim Biophys Acta 2016;1863:2394-412.

6. Moriyama M, Fujimoto Y, Rikimaru S, et al. Mechanism for increased hepatic glycerol synthesis in the citrin/ mitochondrial glycerol-3-phosphate dehydrogenase doubleknockout mouse: Urine glycerol and glycerol 3-phosphate as potential diagnostic markers of human citrin deficiency. Biochim Biophys Acta 2015;1852:1787-95.
7. Saheki T, Inoue K, Ono H, et al. Oral aversion to dietary sugar, ethanol and glycerol correlates with alterations in specific hepatic metabolites in a mouse model of human citrin deficiency. Mol Genet Metab 2017;120:306-316.

8. Lin WX, Zeng HS, Zhang ZH, et al. Molecular diagnosis of pediatric patients with citrin deficiency in China: SLC25A13 mutation spectrum and the geographic distribution. Sci Rep 2016;6:29732.

9. Zhang ZH, Yang ZG, Chen FP, et al. Screening for five prevalent mutations of $S L C 25 \mathrm{~A} 13$ gene in Guangdong, China: a molecular epidemiologic survey of citrin deficiency. Tohoku J Exp Med 2014;233:275-81.

10. Li YC, Ye WJ, Jiang CG, et al. River Valleys Shaped the Maternal Genetic Landscape of Han Chinese. Mol Biol Evol 2019;36:1643-52.

11. Zhang F, Su B, Zhang YP, et al. Genetic studies of human diversity in East Asia. Philos Trans R Soc Lond B Biol Sci 2007;362:987-95.

12. Lu YB, Kobayashi K, Ushikai M, et al. Frequency and distribution in East Asia of 12 mutations identified in the SLC25A13 gene of Japanese patients with citrin deficiency. J Hum Genet 2005;50:338-46.

13. Tabata A, Sheng JS, Ushikai M, et al. Identification of 13 novel mutations including a retrotransposal insertion in SLC25A13 gene and frequency of 30 mutations found in patients with citrin deficiency. J Hum Genet 2008;53:534-45.

14. Song YZ, Zhang ZH, Lin WX, et al. SLC25A13 Gene Analysis in Citrin Deficiency: Sixteen Novel Mutations in East Asian Patients, and the Mutation Distribution in a Large Pediatric Cohort in China. PLoS One 2013;8:e74544.

15. Graffelman J, Jain D, Weir B. A genome-wide study of Hardy-Weinberg equilibrium with next generation sequence data. Hum Genet 2017;136:727-41.

16. Graffelman J, Weir BS. On the testing of Hardy-Weinberg proportions and equality of allele frequencies in males and females at biallelic genetic markers.Genet Epidemiol 2018;42:34-48.

17. Lin JT, Hsiao KJ, Chen CY, et al. High resolution melting analysis for the detection of SLC25A13 gene mutations in Taiwan. Clin Chim Acta 2011;412:460-5.

18. Kikuchi A, Arai-Ichinoi N, Sakamoto O, et al. Simple and rapid genetic testing for citrin deficiency by screening 11 prevalent mutations in SLC25A13. Mol Genet Metab 2012;105:553-8.

19. Wongkittichote P, Sukasem C, Kikuchi A, et al. Screening of SLC25A13 mutation in the Thai population. World J 
Gastroenterol 2013;19:7735-42.

20. Henegariu O, Heerema NA, Dlouhy SR, et al. Multiplex PCR: critical parameters and step-by-step protocol. Biotechniques 1997;23:504-511.

21. Awasthi SP, Chowdhury N, Neogi SB, et al. Development of a multiplex PCR assay for the detection of major virulence genes in Vibrio cholerae including non-O1 and non-O139 serogroups. J Microbiol Methods 2019;157:54-8.

22. Di D, Sanchez-Mazas A. HLA variation reveals genetic continuity rather than population group structure in East Asia. Immunogenetics 2014;66:153-60.

23. Di D, Sanchez-Mazas A, Currat M. Computer simulation of human leukocyte antigen genes supports two main

Cite this article as: Lin WX, Yaqub MR, Zhang ZH, Mao M, Zeng HS, Chen FP, Li WM, Cai WZ, Li YQ, Tan ZY, Sheng W, Li ZM, Tao XL, Li YX, Zhang JP, Han YB, Li Y, Duan WQ, Ye BN, Li YR, Song YZ. Molecular epidemiologic study of citrin deficiency by screening for four reported pathogenic SLC25A13 variants in the Shaanxi and Guangdong provinces, China. Transl Pediatr 2021;10(6):1658-1667. doi: 10.21037/tp$21-58$ routes of colonization by human populations in East Asia. BMC Evol Biol 2015;15:240.

24. Treepongkaruna S, Jitraruch S, Kodcharin P, et al. Neonatal intrahepatic cholestasis caused by citrin deficiency: prevalence and SLC25A13 mutations among Thai infants. BMC Gastroenterol 2012;12:141.

25. Chen R, Wang XH, Fu HY, et al. Different regional distribution of SLC25A13 mutations in Chinese patients with neonatal intrahepatic cholestasis. World J Gastroenterol 2013;19:4545-51.

26. Oh SH, Lee BH, Kim GH, et al. Biochemical and molecular characteristics of citrin deficiency in Korean children. J Hum Genet 2017;62:305-7. 